\title{
Americium-241 integral radiative capture cross section in over-moderated neutron spectrum from pile oscillator measurements in the Minerve reactor
}

\author{
Benoit Geslot ${ }^{1, \text { a }}$, Adrien Gruel ${ }^{1}$, Paul Ros ${ }^{1}$, Patrick Blaise ${ }^{1}$, Pierre Leconte ${ }^{2}$, Gilles Noguere ${ }^{2}$, Ludovic Mathieu ${ }^{3}$, \\ David Villamarin ${ }^{4}$, Vicente Becares ${ }^{4}$, Arjan Plompen ${ }^{5}$, Stefan Kopecky ${ }^{5}$, and Peter Schillebeeckx ${ }^{5}$ \\ 1 CEA, DEN, DER/SPEx, Cadarache, 13108 Saint-Paul-lez-Durance, France \\ 2 CEA, DEN, DER/SPRC, Cadarache, 13108 Saint-Paul-lez-Durance, France \\ 3 CENBG, CNRS/IN2P3, Université de Bordeaux, Chemin du Solarium, BP. 120, 33175 Gradignan, France \\ ${ }^{4}$ CIEMAT - Nuclear Fission Division - Avenida Complutense, 40, 28040 Madrid, Spain \\ 5 EC-JRC, 2440 Geel, Belgium
}

\begin{abstract}
An experimental program, called AMSTRAMGRAM, was recently conducted in the Minerve low power reactor operated by CEA Cadarache within the frame of the CHANDA initiative (Solving CHAllenges in Nuclear Data). Its aim was to measure the integral capture cross section of ${ }^{241} \mathrm{Am}$ in the thermal domain. Motivation of this work is driven by large differences in this actinide thermal point reported by major nuclear data libraries. The AMSTRAMGRAM experiment, that made use of well characterized EC-JRC americium samples, was based on the oscillation technique commonly implemented in the Minerve reactor. First results are presented and discussed in this article. A preliminary calculation scheme was used to compare measured and calculated results. It is shown that this work confirms a bias previously observed with JEFF-3.1.1 $(\mathrm{C} / \mathrm{E}-1=-10.5 \pm 2 \%)$. On the opposite, the experiment is in close agreement with ${ }^{241} \mathrm{Am}$ thermal point reported in JEFF-3.2 $(\mathrm{C} / \mathrm{E}-1=0.5 \pm 2 \%)$.
\end{abstract}

\section{Introduction}

For nearly 10 years, several experiments have been conducted to improve ${ }^{241} \mathrm{Am}$ radiative capture cross section. Indeed, evaluated cross sections from major libraries differ from $10 \%$ to $15 \%$, especially in the thermal domain [1-3]. For instance, a difference of $9 \%$ on the thermal point can be observed between JEFF-3.2 and ENDF/B-VII.1 nuclear data libraries. An international collaborative working group organized by NEA-OECD started in 2015 with the aim of explaining such discrepancies [4].

In this framework, an experimental program was recently conducted by CEA Cadarache in the Minerve reactor. Its main objective was to measure ${ }^{241} \mathrm{Am}$ integral capture cross section in a very thermal neutron spectrum, providing valuable information on the ${ }^{241} \mathrm{Am}$ thermal point, with a target accuracy of $3 \%(1 \sigma)$.

This experiment made use of 7 americium samples manufactured by ITU Karlsruhe in the framework of a previous collaboration between EC-JRC and CEA [5]. Measurements setup was based on the reactivity oscillator technique commonly implemented in Minerve [7]. Gold and lithium samples with the same geometry as americium samples were used for calibration.

\section{Experimental setup}

\subsection{Reactor configuration}

Minerve is a pool type reactor operated at low power ( $100 \mathrm{~W}$ maximum). At the center of the experimental zone, an irradiation channel makes it possible to introduce various material samples in the core. Samples are held in an oscillator that is used to perform pile oscillator experiments. Samples reactivity worth (in the range of a few $\mathrm{pcm}$ ) are obtained and compared against reference materials.

In the AMSTRAMGRAM configuration, a water hole is arranged at the center of the experimental zone. Its dimensions (roughly $4.4 \mathrm{~cm}$ in radius) were chosen so as to maximize the moderation ratio at the oscillation location (see Fig. 1).

The neutron energy spectrum in the oscillation channel exhibits a strong thermal component, as shown on Fig. 2. Proportions of neutrons falling into four energy groups from thermal (below $0.625 \mathrm{eV}$ ) to fast (above $1 \mathrm{MeV}$ ) are indicated in percentage. The cumulative capture reaction rate is also plotted (dotted curve). It shows that the thermal domain (below the first resonance at $0.3 \mathrm{eV}$ ) accounts for around $80 \%$ of the integral capture rate.

\subsection{Americium samples}

Americium samples used in this work were manufactured by ITU Karlsruhe from raw material given by CEA Marcoule. Some of them were previously irradiated at

\footnotetext{
a e-mail: benoit.geslot@cea.fr
}

(c) The Authors, published by EDP Sciences. This is an Open Access article distributed under the terms of the Creative Commons Attribution License 4.0 (http://creativecommons.org/licenses/by/4.0/). 


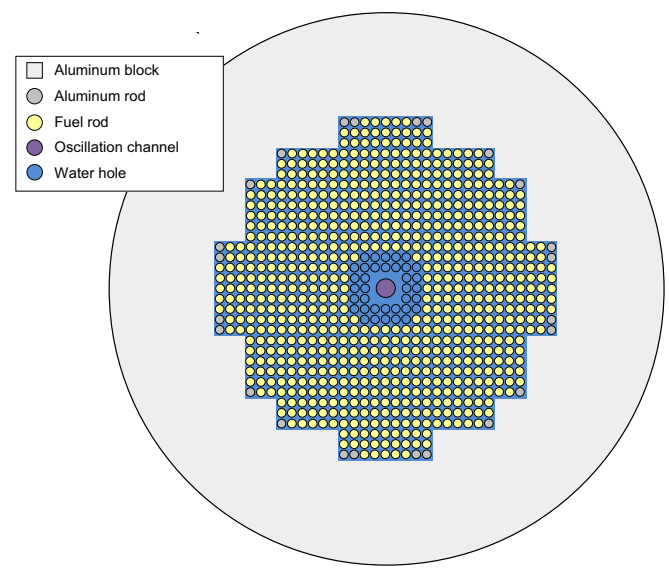

Figure 1. Drawing of the AMSTRAMGRAM experimental zone.

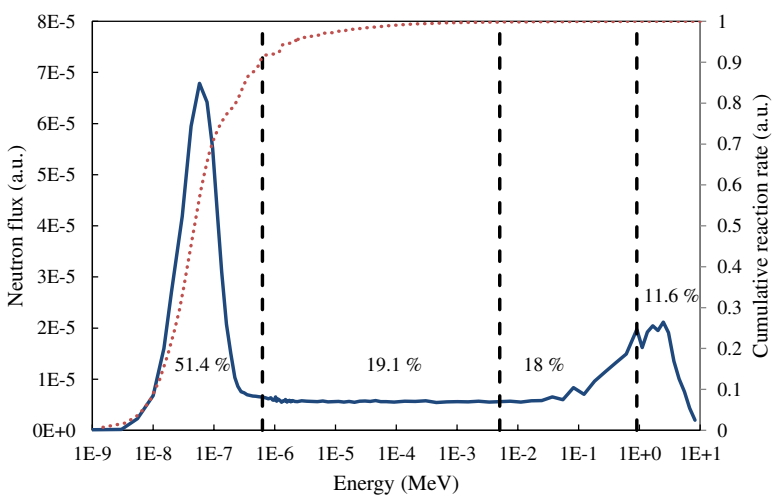

Figure 2. Neutron spectrum in the oscillation channel (solid curve) and cumulative reaction rate related to ${ }^{241} \mathrm{Am}$ capture cross section (dotted line).

EC-JRC for the measurement of the ${ }^{241} \mathrm{Am}(\mathrm{n}, 2 \mathrm{n})$ cross section [5] and at the $n$ ToF facility at CERN for radiative capture measurements [6].

Samples are made of ${ }^{241} \mathrm{AmO}_{2}$ and $\mathrm{Al}_{2} \mathrm{O}_{3}$ powders mixed and pressed into pellets or $12 \mathrm{~mm}$ in diameter and $2 \mathrm{~mm}$ thickness and then encapsulated in aluminum containers. Americium mass is around $40 \mathrm{mg}$ diluted in roughly $400 \mathrm{mg}$ of alumina.

\subsection{Reference samples}

Calibration of the measurement process is obtained by using disks of reference material in dummy aluminium containers. Gold disks $(\varnothing 10 \mathrm{~mm}, 0.125 \mathrm{~mm}$ thick) and lithium disks ( $\varnothing 10 \mathrm{~mm}, 0.2 \mathrm{~mm}$ thick) of high purity were purchased for that purpose.

Additional samples loaded with $400 \mathrm{mg}$ alumina pellets were also measured in order to obtain the reactivity per gram of the alumina matrix.

\section{Data processing}

\subsection{The reactivity oscillator technique}

Oscillation techniques are based on creating a weak and localized perturbation in a characterized neutron flux. Its characteristics (amplitude, neutron spectrum) can be connected to physical parameters of the perturbation source (i.e. isotopic cross sections).
Oscillation techniques can be classified in function of three main parameters: use of a power compensation system, the oscillator frequency, the detector location. Detailed information can be found in the literature [7].

The technique performed in Minerve is called "reactivity oscillator". It is based on the compensation of the reactivity perturbation induced by the sample motion in the core. The reactor is kept critical using a rotating cadmium rod, which is driven by a monitor boron chamber, located in the reflector, through a retro-active loop.

The measured signal is given by the cadmium rod rotation angle. It is proportional to the small sample reactivity worth. A simple way to understand it is to express the reactivity of reactor states using the exact perturbation theory. Let 1 be a reactor state perturbed by a small reactivity sample and 0 be the reference state. $P$ and $L$ refer to operators of production and absorption, $\lambda=1 / k$ where $k$ is the multiplication factor and $\Psi$ and $\Psi^{+}$are forward and adjoint fluxes. Then, the reactivity difference between the two reactor states can be expressed as:

$$
\delta \rho=\frac{\left\langle\Psi_{0}^{+} \mid\left(\lambda_{0} \delta P-\delta L\right) \Psi_{1}\right\rangle}{\left\langle\Psi_{0}^{+} \mid P_{1} \Psi_{1}\right\rangle} .
$$

Making the assumption that the test sample is mainly an absorber, it gives:

$$
\delta \rho \approx \frac{\left\langle\Psi_{0}^{+} \mid-\delta L \Psi_{1}\right\rangle}{\left\langle\Psi_{0}^{+} \mid P_{0} \Psi_{1}\right\rangle} .
$$

It is then possible to express reactivity ratios against reference materials, like gold or lithium:

$$
\frac{\delta \rho}{\delta \rho_{r e f}}=\frac{\iint \Psi_{0}^{+} \sigma(E) \Psi_{1} d r d E}{\iint \Psi_{0}^{+} \sigma_{r e f}(E) \Psi_{r e f} d r d E} .
$$

Introducing the adjoint weighted absorption factor:

$$
f(\sigma)=\frac{\iint \Psi_{0}^{+} \sigma(E) \Psi_{1} d r d E}{\iint \sigma(E) \Psi_{1} d r d E},
$$

Eq. (3) can be rewritten as follows:

$$
\frac{\delta \rho}{\delta \rho_{r e f}}=\frac{f(\sigma)}{f\left(\sigma_{r e f}\right)} \times \frac{\iint \sigma(E) \Psi_{1} d r d E}{\iint \sigma_{r e f}(E) \Psi_{r e f} d r d E} .
$$

In the previous equation, the left hand side term is directly obtained from the experiment. The right hand side term is divided into two factors. The first one can be calculated using a deterministic code like APOLLO2 in a $2 \mathrm{D}$ geometry and account for a correction of a few percents. The second one can be precisely calculated from a Monte Carlo core calculation.

\subsection{Measurement setup}

An americium sample has a reactivity worth of $-0.2 \mathrm{pcm}$ roughly, which is quite low compared to the measurement range $(\sim 10 \mathrm{pcm})$. In order to increase the signal-to-noise ratio of the measurement, several samples were oscillated at the same time. For that purpose, up to 5 americium samples (and up to 7 gold samples) were loaded columnwise in a sample container. The container was made of aluminum alloy 5754 and sealed by screw-in plugs. It was 
placed in an oscillator tube along with aluminum rods (al 5754 as well) so that its mid-plane was aligned with the core mid-plane when the oscillator was in its lower position.

Measurements were composed of sets of 5 oscillation cycles with $120 \mathrm{~s}$ period. Acquisition was trigged by the oscillator control system and recorded data included 3 analog signals: oscillator position, current of the boron chamber and angle of the cadmium rod versus time.

Every measurement gave 5 values of the rod angle difference between upper and lower position of the sample in the core. For each oscillator loading, measurements were reproduced at least 5 times. If results were consistent (based on a $\chi^{2}$ test), measurements were averaged to obtain a mean value.

To extract the reactivity worth of an isotope of interest, it is necessary to subtract any signal coming from the matrix (alumina) and the container (clad and aluminum wedges). Indeed, at first order, the overall reactivity can be linearly divided into a sum of individual components:

$$
\delta \rho_{\text {sample }}=\delta \rho_{A m}+\delta \rho_{\text {alu }}+\delta \rho_{\text {clad }}
$$

Last terms in Eq. (6) can be obtained by oscillating dedicated samples (empty clads or samples loaded with alumina only). Corrections should take into account any significant mass differences between samples.

\subsection{Uncertainties management}

Sources of uncertainties that affect the measurement are numerous and difficult to model. They come from mechanical fluctuation in the oscillator, electronic perturbation in signal recording, core evolution during the program, etc. An empirical approach is used to estimate two overall sources of uncertainties: one responsible for variation amongst oscillation cycles, referred to as "repeatability", and another one responsible for any fluctuation from one measurement to the other, called "reproducibility". Those uncertainty sources are supposed to be independent from signal level. Measurements are also assumed to be uncorrelated.

Let $p$ be the number of oscillation cycles per measurement, $j$ be a cycle index, $i$ be a measurement index, $\varepsilon$ be a sample index and $N$ be the total number of measurements in the experiment. $S_{i j}(\varepsilon)$ is then the signal recorded for sample $\varepsilon$ of cycle $j$ in measurement $i$. $S_{i}(\varepsilon)$ is the ensemble average of $n_{\varepsilon}$ measurements of sample $\varepsilon$. The repeatability can be estimated as the intra-class variance of signals $S_{i j}(\varepsilon)$ and the reproducibility can be viewed as the inter-class variance of the whole measurements $S_{i}(\varepsilon)$.

$$
\begin{aligned}
\hat{\sigma}_{e}^{2} & =\frac{1}{p \cdot N-1} \sum_{i, j, \varepsilon}\left(S_{i j}(\varepsilon)-\bar{S}_{i}(\varepsilon)\right)^{2} \\
\hat{\sigma}_{c}^{2} & =\frac{1}{N-1} \sum_{\varepsilon} \sum_{i}\left(\bar{S}_{i}(\varepsilon)-\bar{S}(\varepsilon)\right)^{2} \\
\bar{S}(\varepsilon) & =\frac{1}{n_{\varepsilon} p} \sum_{i, j} S_{i j}(\varepsilon)=\frac{1}{n_{\varepsilon}} \times \sum_{i} \bar{S}_{i}(\varepsilon)
\end{aligned}
$$

The final uncertainty affecting the reactivity worth of sample $\varepsilon$ from $n_{\varepsilon}$ measurements of $p$ cycles is expressed as:

$$
\hat{\sigma}_{\varepsilon}=\sqrt{\frac{\hat{\sigma}_{e}^{2}}{n_{\varepsilon} \cdot p}+\frac{\hat{\sigma}_{c}^{2}}{n_{\varepsilon}}} .
$$

\subsection{Experimental results}

Raw signals (in angular unit) are converted into reactivity unit (cent) using a calibration coefficient. It is obtained by measuring the core reactivity (by reactor doubling time) at various cadmium rod angular positions. This coefficient may be somehow imprecise, but it should be noted that it has no impact on reactivity ratios.

Results are given in the Table 1 . The reactivity per unit mass is expressed in cent/g, where 1 cent refers to a reactivity worth equal to one hundredth of the reactor delayed neutron fraction.

The value given for ${ }^{241} \mathrm{Am}$ is obtained by subtracting the reactivity from the alumina matrix, which amplitude is about $2 \%$ (positive) of the overall signal (negative).

When several samples were piled in a sample container, a small shadow effect between samples was observed in the case of lithium and gold. This effect was corrected by extrapolating the reactivity per unit mass measured for different sample loadings down to the average sample mass. Extrapolation was done thanks to a fitting procedure based on a Monte Carlo algorithm sampling the input parameters over Gaussian distribution (correlation on samples masses were taken into account). Uncertainties given in Table 1 (at 1 sigma) are standard deviation calculated over 10000 simulations.

Final mass reactivity ratios are $6.29 \pm 0.07$ in the case of americium / gold and $0.354 \pm 0.005$ in the case of americium / lithium.

\section{Preliminary feedback on nuclear data}

\subsection{Calculation route}

A preliminary analysis of these experiments was performed with the TRIPOLI4-DEV Monte-Carlo code using point-wise cross sections from JEFF-3.1.1 and JEFF-3.2. Although it is possible to use the approximated equations developed in 3.1 in order to compute the reactivity worth and obtain the bilinear integral as described in Eq. (1), we have used a more precise collisionbased exact perturbation method calculation route recently developed [12].

The geometrical model consists of a full detailed 3D heterogeneous description of the core, including graphite reflector, driver zone, test zone and sample inserted the central guide tube.

A calculation was run for each sample kind, based on the average mass given in Table 1. Computing was parallelized over 512 CPUs during $24 \mathrm{~h}$, in order to reduce the uncertainty on the sample reactivity worth below $1 \%(1 \sigma)$. The calculation method provides the overall reactivity worth, as well as an uncertainty breakdown over isotopes, reactions and energy groups.

\subsection{Preliminary calculated results}

A clear trend can be derived from this preliminary analysis. An $11 \%$ difference is obtained in the $\mathrm{C} / \mathrm{E}-1$ value when comparing calculation using nuclear data libraries JEFF3.1.1 and JEFF-3.2 (Table 2). This confirms a similar trend 
Table 1. Reactivity per unit mass for test materials.

\begin{tabular}{|l|l|l|l|}
\hline Material & $\begin{array}{l}\text { Mass } \\
(\mathrm{mg})\end{array}$ & $\begin{array}{l}\text { Mass reactivity } \\
(\text { cent/g) }\end{array}$ & $\begin{array}{l}\text { Uncertainty } \\
(\text { cent/g) }\end{array}$ \\
\hline $\mathrm{Al}_{2} \mathrm{O}_{3}$ & 396 & 0.009 & $1.710^{-3}$ \\
\hline${ }^{197} \mathrm{Au}$ & 185.4 & -0.708 & $510^{-3}$ \\
\hline${ }^{\text {nat }} \mathrm{Li}$ & 8.38 & -12.5 & 0.15 \\
\hline${ }^{241} \mathrm{Am}$ & 41.5 & -4.46 & $3.4 \times 10^{-2}$ \\
\hline
\end{tabular}

Table 2. Calculation over experiment for JEFF-3.1.1 and JEFF-3.2. Uncertainty is given at $1 \sigma$.

\begin{tabular}{|l|c|c|c|}
\hline Library & $\begin{array}{c}\text { Thermal } \\
\text { point (b) }\end{array}$ & $\begin{array}{c}\text { Resonance } \\
\text { Integral (b) }\end{array}$ & C/E - 1 (\%) \\
\hline JEFF-3.1.1 & 647 & 1526 & $-10.5 \pm 2$ \\
\hline JEFF-3.2 & 747 & 1826 & $0.5 \pm 2$ \\
\hline
\end{tabular}

observed in UOx-PWR and MOx-PWR spectra obtained from the OSMOSE program performed in Minerve [8,9].

Moreover, preliminary calculations based on one container loaded with 7 americium samples with JEFF3.2 with a $\mathrm{C} / \mathrm{E}$ value very close to 1 ( $0.5 \%$ difference). Since reactivity sensitivity to ${ }^{241}$ Am capture cross section is around 0.8 for the thermal domain, that result supports very closely the ${ }^{241} \mathrm{Am}$ thermal point value as reported in JEFF-3.2.

Currently, the uncertainty on the $\mathrm{C} / \mathrm{E}-1$ value only includes measurement uncertainty and MonteCarlo statistical uncertainty but it does not include any technological uncertainties (sample and fuel pin composition and dimensions). The final uncertainty should be typically in the range of $3 \%$ to $4 \%$.

\section{Conclusions}

This paper presents preliminary experimental results from the AMSTRAMGRAM program conducted by CEA in the Minerve reactor, in collaboration with EC-JRC. A very thermal neutron spectrum was designed for the experiment that aimed at providing a new integral measurement of ${ }^{241}$ Am radiative capture cross section with a target uncertainty of less than $3 \%$. The experiment, based on the pile oscillator technique, was only possible thanks to EC-JRC that provided several well characterized americium samples. Preliminary analysis shows a clear trend when comparing measurements to calculations associated to JEFF-3.1.1 and JEFF-3.2 libraries. First results are in very good agreement with JEFF-3.2 thermal point with a preliminary discrepancy of $0.5 \pm 2 \%$.

This work was conducted in the framework of the CHANDA project, which is part of the European Commission $7^{\text {th }}$ Framework Programme. The collaboration regroups CIEMAT, EC-JRC, CNRS and CEA. The authors are thankful to EDF for supporting and funding part of the AMSTRAMGRAM experiment.

\section{References}

[1] C. Lampoudis, S. Kopecky, O. Bouland, F. Gunsing, G. Noguere, A.J.M. Plompen, C. Sage, P. Schillebeeckx, R. Wynants, Eur. Phys. J. Plus 128 (2013)

[2] G. Noguere, O. Bouland, S. Kopecky, C. Lampoudis, P. Schillebeeckx, A. Plompen, F. Gunsing, C. Sage, and I. Sirakov, Phys. Rev. C 92, 014607 (2015)

[3] D. Bernard, O. Bouland, J. Nucl. Sci. Tech. 49 (2012)

[4] www.oecd-nea.org/science/wpec/sg41

[5] C. Sage, V. Semkova, O. Bouland, P. Dessagne, A. Fernandez, F. Gunsing, C. Nästren, G. Noguère, H. Ottmar, A.J.M. Plompen, P. Romain, G. Rudolf, J. Somers, F. Wastin, Phys. Rev. C 81, 064604 (2010)

[6] K. Fraval, F. Gunsing, et al., Physical Review C 89(4), 044609 (2014)

[7] W.K. Foell, "Small-sample reactivity measurements in nuclear reactors", American Nuclear Society, Hinsdale, IL, USA (1972)

[8] M. Antony, J. Di-Salvo, A. Pepino, J.-C. Bosq, D. Bernard, P. Leconte, A. Lyoussi, Proceedings of 1 st Int. Conf. on Advancements in Nuclear Instrumentation, Measurement Methods and their Applications, Marseille, France (2009)

[9] D. Bernard, P. Leconte, A. Gruel, M. Antony, A. Pepino, J. Di Salvo, A. Lecluze, J.-F. Ledoux, C. Morel, B. Sabatin, Journal of the Korean Physical Society 59, 23 (2011)

[10] R. Sanchez, I. Zmijarevic, "APOLLO2 Year 2010”, Nuclear Engineering and Technology 42(5), October (2010)

[11] J.-P. Both, Proc. Int. Conf. Supercomputing in Nuclear Applications (SNA2003), Paris, France, September 22-24, 2003

[12] G. Truchet, P. Leconte, A. Santamarina, et al., Proc. Int. Conf. SNA+MC2013, La Cité des Sciences et de l'Industrie, Paris, France, October 27-31 (2013) 